\title{
Study of the Side effects profile of different antihypertensive drugs among the Hypertensive patient
}

\author{
Shrestha $\mathrm{RK}^{1}$, Khan GM${ }^{1}$, Thapa $\mathrm{P}^{1}$, Koju $\mathrm{R}^{2}$ \\ ${ }^{1}$ Department of Pharmacy, Kathmandu University \\ ${ }^{2}$ Department of Medicine, Dhulikhel Hospital
}

\begin{abstract}
Background

Hypertension is the leading cause of morbidity and mortality worldwide. Because hypertension is usually asymptomatic yet requires long term therapy, consideration of potential of undesirable effects of drugs used for its treatment is important for making the appropriate choice. In this context, a precise understanding typical adverse reaction profile of the different drugs is essential.
\end{abstract}

\section{Objective}

This study was done to assess the side effects profile of different antihypertensive drugs (calcium channel blocker, beta blocker, ACE inhibitor) among the hypertensive patient.

\section{Methods}

A mixed retrospective \& prospective cross sectional study of 61 patients was done at Medical Out Patient Department of Dhulikhel Hospital KUTH were included in the study.

Result

Dry cough was experienced by most of the patients (66.7\%) and both dry cough and dizziness was reported by only one patient (6.7\%) taking enalapril. In case of atenolol, bradycardia, dizziness and insomnia were experienced by same number of patients (14.3\%) separately. Among amlodipine users, side effects experienced were peripheral edema (23\%), flushing (2.6\%), dyspnoea (2.6\%), headache (5.1\%), dizziness (2.6\%), palpitation (2.6\%) and insomnia (2.6\%).Patients on enalapril had significantly more complaints regarding side effects $(73.3 \%)$ compared to those taking amlodipine $(41 \%)(\mathrm{OR}=4, p=0.033)$ and atenolol $(42.9 \%)$.There was no significant difference in the occurrence of side effects between enalapril- atenolol $(p>0.05)$ and amlodipine- atenolol $(p>0.05)$.

\section{Conclusion}

Although all three drugs were well tolerated, more side effects were seen in case of enalapril. Enalapril has 4 times higher rate of side effects than amlodipine.

Key word Antihypertensive drugs, Side effects, Enalapril, amlodipine. 


\section{Introduction}

Hypertension is the leading cause of morbidity and mortality worldwide. It is a potent risk factor for second major cardiovascular events, but the most for cerebrovascular accidents particularly in the elderly. ${ }^{1}$ The World Health Organization (WHO) report which examined the major risk factors for global disease identified hypertension as one of the most important causes of the disease burden of developed and developing nations. ${ }^{2}$

Because hypertension is usually asymptomatic yet requires long-term therapy, consideration of potential undesirable effects of drugs used for its treatment is important for making the appropriate choice. In this context, a precise understanding of the efficacy and typical adverse reaction profiles of the different drugs is essential. Even with a good knowledge of all of these aspects, however, no fixed plan for drug treatment of hypertension suitable for every patient can be established. A good control of blood pressure and avoidance of adverse drug reactions can, however, be achieved by appropriate drug selection and dosage, carried out after careful consideration of the known adverse reaction profiles as well as the known spectrum of pharmacological actions of the different compounds in reducing blood pressure.

The side effects of different antihypertensive medication are also not well studied in Nepalese population despite of clearly mentioned in textbook and literature. The result on side effect profile would provide the necessary information on side effect of different antihypertensive medications as well as some information on reasons of poor compliance of antihypertensive medication.

\section{Methods}

This study was approved by Institutional Review Committee, Kathmandu University School of Medical Sciences. A total of sixty one patients who had been taking antihypertensive as monotherapy or newly diagnosed hypertensive patients who were prescribed antihypertensive were included in this study. This study was done by reviewing all available documents and interviewing the patient during regular hospital visits. The study included data available several years ago to till latest available data during study period. Those newly diagnosed patients were followed up as possible. Clinical information and details regarding side effects were collected from those patients at medical outpatient department of Dhulikhel Hospital, KUTH from $3^{\text {rd }}$ December, 2006 to $29^{\text {th }}$ April, 2007. Patient taking other than antihypertensive medication, patient having other comorbid condition like IHD, heart failure, COPD, diabetic mellitus, peripheral vascular disease, cardiomyopathy \& others were not included in the study.

Preliminary questionnaire was prepared and filed tested for 1 week then it was modified as per requirement accordingly. Data from interview were collected by using questionnaire. Past treatment \& clinical details were obtained from the medical records.

After taking written consent from the patient, the information was obtained. They were asked about side effects which they felt after taking medication. This entire information gathered was entered in prepared questionnaire. All date collected were coded as per variables and entered in SPSS data sheet and analyzed using the statistical software SPSS 13.0 for windows. The significant differences of side effect between drugs were analyzed using $\chi^{2}$ test. $P$-value of $<0.05$ was taken as statistically significant.

\section{Results}

The details of use of medication are shown in Table 1. Among 61 patients, amlodipine was being used by 39 patients whereas enalapril and atenolol were being used by 15 and 7 patients respectively. Among amlodipine users, nearly fifty percent of patients were of age group of 3050 yrs. About more than half $(56.1 \%)$ of patients using amlodipine were female.

In case of enalapril, near about two third (73.3\%) were female and one third (26.7\%) were male. Enalapril was found to be mostly (46.7\%) used by patient of age group of 40-50 yrs.

Among the atenolol users, most (57.1\%) of the patients were of age group 40-50yrs. Females (73.3\%) were predominantly using atenolol than male (28.6\%).

Table 1. Details of Use of Medication in 61 Patients

\begin{tabular}{|lcccc|}
\hline Characteristics & & Amlodipine & Enalapril & Atenolol \\
\hline Age & & & & \\
\hline & $<20$ yrs & $1(2.4)$ & & \\
& $20-30 y r s$ & $2(5.1)$ & $2(13.3)$ & \\
\hline & $30-40$ yrs & $10(25.5)$ & $1(6.7)$ & $1(14.3)$ \\
\hline & $40-50$ yrs & $10(25.5)$ & $7(46.7)$ & $4(57.1)$ \\
\hline & $50-60$ yrs & $6(15.3)$ & $1(6.7)$ & $1(14.3)$ \\
\hline & $60-70$ yrs & $7(17.7)$ & $2(13.3)$ & $1(14.3)$ \\
\hline Total no of patients & $>70$ yrs & $3(7.5)$ & $2(13.3)$ & \\
\hline Gender & & 39 & 15 & 7 \\
\hline & Male & $17(43.9)$ & $4(26.7)$ & $2(28.6)$ \\
\hline & Female & $22(56.1)$ & $11(73.3)$ & $5(71.4)$ \\
\hline
\end{tabular}


The side-effects of three categories of antihypertensive drugs reported by the patients on mono-therapy are shown in Table 2. Among amlodipine users, $41 \%$ of patient reported side effects. Patients on enalapril complained of side effects more frequently $(73.3 \%)$ than other class of drugs whereas $42.9 \%$ of patients on atenolol experienced side effects.

It was found that among 15 patients taking enalapril, 10 (66.7\%) patients experienced dry cough and 1 (6.7\%) experienced both dry cough and dizziness. In case of atenolol, 1 patient experienced bradycardia, 1 patient experienced dizziness and 1 patient experienced insomnia whereas among 39 patients taking amlodipine,9 (23\%) patient experienced peripheral edema,1 (2.6\%)-flushing, 2 (5.1\%)- headache,1(2.6\%)-dizziness, 1 (2.6\%)- palpitation, 1 (2.6\%)- dyspnoea, 1 (2.6\%)- insomnia.

Table 2. Details of Side -effects

\begin{tabular}{llcc|}
\hline & $\begin{array}{c}\text { Amlodipine } \\
(\mathbf{n}=39)\end{array}$ & $\begin{array}{c}\text { Enalapril } \\
(\mathbf{n = 1 5})\end{array}$ & $\begin{array}{c}\text { Atenolol } \\
(\mathbf{n}=\mathbf{7})\end{array}$ \\
\hline Side -effect & $16(41)$ & $11(73.3)$ & $3(42.9)$ \\
\hline $\begin{array}{l}\text { Types of side -effect seen } \\
\text { Peripheral edema }\end{array}$ & $9(23)$ & & \\
\hline Drycough & & $10(66.7)$ & \\
\hline Bradycardia & & & \\
\hline Flushing & $1(2.6)$ & & $1(14.3)$ \\
\hline Headache & $2(5.1)$ & & \\
\hline Dizziness & $1(2.6)$ & & $1(14.3)$ \\
\hline Palpitation & $1(2.6)$ & & \\
\hline Dyspnoea & $1(2.6)$ & & $4(57.1)$ \\
\hline Insomnia & $1(2.6)$ & & $1(6.6)$ \\
\hline Dry Cough with Dizziness & & $4(26.7)$ & \\
\hline No Side-effects & $23(58.9)$ & & \\
\hline
\end{tabular}

There was statistically significant difference in the occurrence of side effects between drug amlodipine and enalapril ( $p=0.033)$. The risk of occurring side effect with enalapril was found four times higher than amlodipine. But there was no significant difference in the occurrence of side-effects between drug enalapril - atenolol ( $p=0.343$ ) and amlodipine - atenolol $(p=1)$

Table 3. Details of comparison between two medication

\begin{tabular}{|l|c|c|c|c|}
\hline & Side effects & No side effects & p-value & OR \\
\hline Amlodipine & $16(41)$ & $23(59)$ & & \\
\hline Enalapril & $11(73)$ & $4(26.7)$ & 0.033 & 4 \\
\hline
\end{tabular}

\begin{tabular}{|l|c|c|c|}
\hline & Side effects & No side effects & p-value \\
\hline Enalapril & $11(73.3)$ & $4(26.7)$ & \\
\hline Atenolol & $3(42.9)$ & $4(57.1)$ & 0.343 \\
\hline
\end{tabular}

\begin{tabular}{|l|c|c|c|}
\hline & Side effects & No side effects & p-value \\
\hline Amlodipine & $16(41)$ & $23(59)$ & \\
\hline Enalapril & $3(42.9)$ & $4(57.1)$ & 1 \\
\hline
\end{tabular}

\section{Discussion}

Regarding the use of drugs, amlodipine was the most frequently prescribed drug. This might be due to less complication of CCBs, high safety of amlodipine and preferences in case of treatment of hypertension with no previous treatment. British HTN society guidelines recommended use of either [ACEI or BB] or [CCB or diuretics] as the first line antihypertensives. Angiotensin converting enzyme inhibitor and calcium channel blockers are recommended by the JNC VI as alternative to diuretics and $\beta$-blocker unless compelling indications for the first line use are present. Despite these recommendations, ACE inhibitors and calcium channel blockers are consistently the most frequently prescribed antihypertensive agents in both younger and older patients with hypertension. ${ }^{3}$

The usefulness of antihypertensive drugs depends not only on the degree to which blood pressure is lowered but also on the side effects profile. Side effects of antihypertensive medication affect tolerability and compliance. So they are becoming more important issue.

In this study, all three drugs were reasonably well tolerated. The side effects experienced most frequently in amlodipine were peripheral edema $(23 \%, n=9)$, headache $(5.1 \%, n=2)$, flushing $(2.6 \%, n=1)$, dizziness $(2.6 \%, n=1)$, palpitation $(2.6 \%, n=1)$, dyspnoea $(2.6 \%, n=1)$, insomnia $(2.6 \%, n=1)$. Edema occurs with CCBs because of vasodilation in the distal arterioles, thereby leading to increased intravascular capillary pressures and increased venous pressures, at least in the lower extremities and eventually leakage of fluid into the extracellular space. ${ }^{4}$ Excessive vasodilatation results in headache, dizziness, flushing etc.

The side effects experienced by enalapril were dry cough $(66.7 \%, n=10)$ and dry cough with dizziness $(6.6 \%, n=1)$. The cough is typically irritating, dry and nonproductive and is not dose related. In 5 to $20 \%$ of patients, ACE inhibitors induce a bothersome, dry cough. A number of suggestions have been put forward as to the etiology of the cough including potentiation of bradykinin levels by ACE inhibition, an increased sensitivity of the normal cough reflex, and finally increased levels of circulating prostaglandins. However, the cough is definitely due to inhibition of ACE rather than the 
blockade of the renin-angiotensin system. The percentage of side effects is more in this study. It may be due to the fact that the patients attending outpatient department are mostly due to problems related with either uncontrolled blood pressure or side effects of drugs.

The side effects experienced with atenolol were bradycardia $(14.3 \%, \mathrm{n}=1)$, palpitation $(14.3 \%, \mathrm{n}=1)$ and insomnia $(14.3 \%, n=1)$.Atenolol is a cardio selective drug and potent in blocking $\beta_{1}$-receptors.the blockade of this receptors decreases the heart rate and result bradycardia. ${ }^{5}$

Patients on enalapril had significantly more complaints regarding side effects than other categories of drugs. The risk of occurring side effect with enalapril was 4 times higher than amlodipine. This result is similar to the study of Gryglas $\mathrm{P}^{6}$ in which more side effects were experienced in enalapril group than that of amlodipine. Similar result was obtained in study conducted by Webster et al., in which amlodipine was found to be safer than atenolol.

There was no significant difference in the occurrence of side effects between enalapril- atenolol and amlodipine -atenolol. The result obtained is similar to the result obtained in some of previous studies in which amlodipine and atenolol were well tolerated. ${ }^{7}$ After 4 weeks of therapy the incidence of side effects was slightly, but insignificantly, higher on atenolol than on enalapril. ${ }^{8}$ No serious side effects were encountered with enalapril and atenolol. ${ }^{9}$ However, when compared with the beta-blockers atenolol and nadolol, amlodipine had a favorable safety profile. In particular, the incidence of severe side effects in patients receiving amlodipine was approximately half than that reported for patients receiving beta-blockers. ${ }^{10}$ It should be considered that the finding of the present study has to be interpreted with caution as the sample size was limited.

It should be noted that this is a cross-sectional study and hence, the possibility of missing the patients who discontinued the drug or stopped follow-up due to poor
BP control or side -effects cannot be ruled out. Hence, the overall result may not give a realistic picture. A study conducted in the United Kingdom where as many as half of all patients who used antihypertensive drugs have been found to discontinue treatment within follow-up periods ranging from 6 months to 4 years and $42 \%$ of all changes in antihypertensive therapy were due to side effects. ${ }^{11}$ Those reporting problems with their medications were 3.5 times more likely to reduce their doses or discontinue therapy than patients not reporting problems.

The study being retrospective and mono-centered involving limited sample size, the real influence of various parameters with regards to side effects could have been missed. However, this study definitely provides a perspective of side effects profile of the antihypertensive drugs. The findings of this study will need to be revalidated with larger sample size and longer follow-up times to derive stronger conclusions.

\section{Conclusion}

Attention must be paid to side- effects that can cause patient to drop out of treatment and might lead to failure to control hypertension. Calcium channel Blockers, ACE inhibitor and $\beta$-blockers are considered to be the most popular drugs class. In the present study, CCBs were best tolerated. The risk of occurring side effects with enalapril was found 4 times higher than Amlodipine. There was no significant difference in the occurrence of sideeffects between drug enalapril - atenolol $(p=0.343)$ and amlodipine - atenolol $(p=1)$.

Peripheral edema due to amlodipine and dry cough due to enalapril were more common side effects experienced by these drugs respectively. Although all three drugs were well tolerated, more side effects were seen in case of enalapril.

\section{References}

1. Kjeldsen SE, Julius $\mathrm{S}$, Hedner $\mathrm{T}$, et al. Stroke is more common than myocardial infarction in hypertension: analysis based on 11 major randomized intervention trials. Blood Press 2001;10:190-2.

2. Ezzati $M$, Lopez $A D$, Rodgers $A$, et al. Selected major risk factors and global and regional burden of disease. Lancet 2002;360:1347-60.

3. Nelson CR, Knapp DA. Trends in antihypertensive drug therapy of ambulatory patients by US office based physicians. Hypertension 2000;36:600-3.

4. Knight EL, Glynn RJ, Levin R, et al. Failure of evidence-based medicine in the treatment of hypertension in older patients. $J$ Gen Intern Med 2000;15:702-9.

5. Ciccone $M$, DiNoia $D$, DiMichele $L$, et al. Effects of lacidipine and nifedipine on the lower limb veins of nonphlebopathic patients. J Cardiovasc Pharmacol 1994;23:S111-2.

6. Katzung BG, Basic and Clinical Pharmacology, $8^{\text {th }}$ edition. San Francisco:McGraw- Hill. p 155-90 
7. Gryglas P. The comparison of hypotensive efficiency and tolerability of amlodipine and enalapril in patients with essential hypertension. Pol Arch Med Wewn 2001;105(2):10915.

8. Frishman $\mathrm{WH}$, Brobyn $\mathrm{R}$, Brown RD, et al. Amlodipine Versus atenolol in essential hypertension. Am J Cardiol 1994;73(3)50A-54A.

9. Edmonds D. Enalapril as a first-step agent in essential hypertension: a comparative study with atenolol. J Hypertens Suppl 1986 Dec;4(5):S406-9.

10. Mangoush M, Singh NK, Kumar S, et al. Efficacy of enalapril in essential hypertension and its comparison with atenolol. Postgrad Med J 1990 Jun;66(776):446-9.
11. Osterloh I. The safety of Amlodipine. Am Heart J 1989 Nov;118(5 Pt 2):1114-9.

12. Lip GY, Beevers DG. Doctors, nurses, pharmacists and patients - the Rational Evaluation and Choice in Hypertension (REACH) survey of hypertension care delivery. Blood Press Suppl.1997;1:6-10 